Pacific Journal of Mathematics

ON BOREL PRODUCT MEASURES

WiLson BLEDSOE and ChaRLES EDWARD WILK 


\title{
ON BOREL PRODUCT MEASURES
}

\author{
W. W. Bledsoe and C. E. Wilks
}

It has been known for many years that the product of two regular borel measures on compact hausdorff topological spaces may not be borel in the product topology. The problem of defining a new product measure that extends the classical product measure and carries over this borel property has been approached in different ways by Edwards, by Bledsoe and Morse (Product Measures, Trans. Amer. Math. Soc. 79 (1955), 173_215; called PM here.) and by Johnson and Berberian. Godfrey and Sion and Hall have shown that all three of these methods are equivalent for the case of Radon measures on locally compact hausdorff spaces.

Elliott has extended the results of $P M$ by defining a product measure for a pair, the first of which is a (generalized) borel measure and the second a continuous regular conditional measure (generalization of conditional probability), and proving a corresponding Fubini-type theorem.

The purpose of this paper is to extend the results of $\mathrm{PM}$ in a manner similar to Elliott's, but with his continuity condition replaced by an absolute continuity condition and by a "separation of variables" condition. It is still an open question whether Elliott's continuity condition is necessary.

1. Definitions and Notation ${ }^{1}$. By a measure (outer measure) $\mu$ on a space $M$ is meant a nonnegative countably subadditive function on $2^{M}$, the subsets of $M$. In a topological space $(M, m)$, an m-borel measure on $M$ is any measure on $M$ for which the open sets are (Caratheodory) measurable, and the borel sets of $(M, m)$ are the members of the smallest $\sigma$-algebra containing $m$. If $G$ is any family of sets, let $\sigma G$ be the union $\bigcup_{\alpha \in G} a$ of the family $G$. If $H \cong 2^{M}$ and $g$ is a nonnegative function on $H$, then $m s s g M H$ is defined to be the function on $2^{M}$ such that $m s s g M H(A)=\inf _{G} \sum_{a \in G} g(a)$ where $G$ varies over all countable subsets of $H$ for which $A \subseteq \sigma G$. $\psi=m s s g M H$ is called the measure generated by the gauge $g$, and $H$ is called the basis of $\psi$.

2. Product measures. If $\mu$ measures $M$ and $\nu$ measures $N$, we call a subset $D$ of $M \times N$ a nilset (more correctly a $\mu \nu$-nilset) if

$$
\iint C r_{D}(x, y) \mu d x \nu d y=0=\iint C r_{D}(x, y) \nu d y \mu d x,
$$

where $C r_{D}$ is the characteristic function on $D$. The ordinary product measure of $\mu$ and $\nu$ is given by

\footnotetext{
${ }^{1}$ Most of the notation used here is taken from [2] and [4].
} 


$$
\psi=m s s g(M \times N) R
$$

where $R$ is the set of $\mu \nu$-measurable rectangles, and $g(a \times b)=\mu(a) \cdot \nu(b)$ for $a \times b \in R$. The extended product measure of $\mu$ and $\nu$ given in [2] is

$$
\phi=m s s g(M \times N)(R \cup Z)
$$

where $Z$ denotes the set of $\mu \nu$-nilsets, and

$$
g(D)=\iint C r_{D}(x, y) \mu d x \nu d y
$$

for $D \in(R \cup Z)$. (i.e., $g(D)=0$ for $D \in Z$, and $g(a \times b)=\mu(a) \cdot \nu(b)$ for $a \times b \in R)$.

It was shown in [2] that

$\phi$ is an extension of $\psi$,

(i.e., if $A$ is $\psi$-measurable then $A$ is $\phi$-measurable and $\phi(A)=\psi(A)$ ). In cases where the nilsets are $\psi$-measurable then, of course, $\phi$ and $\psi$ are identical; but the $\psi$-measurability of nilsets is still an open question in the interesting case when $\mu$ and $\nu$ are regular borel measures on compact hausdorff spaces.

A result from [2], (Thms. 5.11-5.13), is

Theorem 2.1. If $\mu$ measures $M$ and $\nu$ measures $N$ and $\phi$ is the extended product measure of $\mu$ and $\nu$, then

$.1 \phi$ is an extension of the ordinary product measure of $\mu$ and $\nu$,

.2 u-measurable rectangles are $\phi$-measurable,

.3 uע-nilsets have $\phi$ measure 0 , and

.4 (Fubini) if $f$ is $\phi$-integrable, then

$$
\iint f(x, y) \mu d x \nu d y=\int f(z) \phi d z=\iint f(x, y) \nu d y \mu d x \text {. }
$$

Elliott, in [4], has generalized this result by replacing the measure $\nu$ by a regular conditional measure, defined as follows. Let $\mathscr{R}$ be a family of subsets of $N$ for which $\sigma \mathscr{R} \in \mathscr{R} . \quad \nu$ is called a regular conditional measure on $M \times \mathscr{R}$ if $\nu$ is such a function on $M \times \mathscr{R}$ that

(i) for each $x \in M, \nu_{x}=\nu(x, \cdot)$ is a measure for which members of $\mathscr{R}$ are $\nu_{x}$-measurable, and

(ii) for each $b \in \mathscr{R}$, the function $\nu(\cdot, b)$ is $\mu$-integrable (i.e., $\left.\int \nu(x, b) \mu d x \leqq \infty\right)$.

A rectangle $a \times b$ is a $\mu \mathscr{R}$-basic rectangle if $a$ is $\mu$-measurable, $b \in \mathscr{R}$, and 


$$
\int C r_{a}(x) \nu(x, b) \mu d x<\infty
$$

A set $C \leqq M \times N$ is called a $\mu \nu$-nilset if

$$
\iint C r_{C}(x, y) \nu_{x} d y x=0 \text {. }
$$

The $\mu \nu \mathscr{R}$-product measure (of Elliott) is defined as $m s s g(M \times N) F$, where $F$ is the family consisting of all $\mu 2 \mathscr{R}$-basic rectangles and $\mu \nu$-nilsets, and

$$
g(C)=\iint C r_{C}(x, y) \nu_{x} d y \mu d x
$$

for $C \in F$. A corresponding ordinary (conditional) product measure for $\mu$ and $\nu$ can be defined on $M \times N$ using only $\mu \nu$-basic rectangles [9]. Elliott [4, Thms 1.0, 1.4], generalized 2.1 as follows:

Theorem 2.2. If $\mu$ measures $M, \mathscr{R}$ is a $\sigma$-algebra of subsets of $N, \sigma \mathscr{R}=N \in \mathscr{R}, \nu$ is a regular conditional measure on $M \times \mathscr{R}$, and $\phi$ is the $\mu \nu \mathscr{R}$-product measure, then

$.1 \phi$ is an extension of the ordinary (conditional) product measure of $\mu$ and $\nu$,

$.2 \mu \nu \mathscr{R}$-basic rectangles are $\phi$-measurable,

.3 uע-nilsets have $\phi$-measure 0 , and

.4 (Fubini-like) if $f$ is $\phi$-integrable, then

$$
\int f(z) \phi d z=\iint f(x, y) \nu_{x} d y \mu d x \text {. }
$$

3. Topological measures. Let $(M, m)$ be a topological space with a measure $\mu$ on $M . \mu$ is said to be an $m$-borel measure if members of $m$ (open sets) are $\mu$-measurable. If, additionally, for each $A \in m$,

$$
\mu(A)=\sup _{C} \mu(C),
$$

where $C$ varies over all closed subsets of $A$ for which $\mu(C)<\infty$, then $\mu$ is called an m-inner regular borel measure. $m$ is said to be $\mu$ almost lindelof if for each subfamily $H$ of $m$ for which $M=\sigma H$, and for each $S \subseteq M$ for which $\mu(S)<\infty$, there is a countable subfamily $G$ of $H$ for which $\mu(S-\sigma G)=0$.

A regular conditional measure on $M \times \mathscr{R}$ is said to be $m$-continuous if, for each $b \in \mathscr{R}, \nu(\cdot, b)$ is an $m$-continuous function. that

3.0. Throughout the remainder of this paper we shall assume 
$.1(M, m)$ and $(N, n)$ are topological spaces and that $(P, p)$ is their topological product.

$.2 \mu$ is a finite, ${ }^{2} m$-inner regular borel measure on $M$, and $m$ is $\mu$-almost lindelof. $\nu^{\prime}$ is a finite, ${ }^{2} n$-inner regular borel measure on $N$, and $n$ is $\nu^{\prime}$-almost lindelof.

$.3 \mathscr{R}$ is a $\sigma$-algebra of subsets of $N$ such that $n \leqq \mathscr{R}$.

$.4 \nu$ is a regular conditional measure on $M \times \mathscr{R}$, with the properties that for each $x \in M, \nu_{x}$ is a finite $n$-inner regular borel measure, $n$ is $\nu_{x}$-almost lindelof and each member of $\mathscr{R}$ is $\nu_{x}$-measurable.

It should be noted that conditions 3.0 are satisfied if $(M, m)$ and $(N, n)$ are locally compact hausdorff spaces, and $\mu$ and $\nu_{x}$ are finite regular borel measures on $M$ and $N$ respectively, for $x \in M$.

One of the principal results (Th. 7.7) of [2] is the following:

THEOREM 3.1. If $\mu$ and $\nu^{\prime}$ are measures satisfying conditions 3.0.1 and 3.0.2, and $\phi^{\prime}$ is the extended product measure of $\mu$ and $\nu^{\prime}$, then

$.1 \phi^{\prime}$ is a p-inner regular borel measure on $P$, and

$.2 p$ is $\phi^{\prime}$-almost lindelof.

Elliott, in 2.3 of [4], generalized this result as follows:

THEOREM 3.2. If $\mu$ and $\nu$ satisfy conditions 3.0, and $\phi$ is the $\mu \nu \mathscr{R}$-product measure, and if $\nu$ is m-continuous, then

$.1 \phi$ is a p-inner regular borel measure on $P$, and

$.2 p$ is $\phi$-almost lindelof.

The continuity condition of 3.2 can be replaced by an absolute continuity condition, as follows:

THEOREM 3.3. If $\mu, \nu$, and $\nu^{\prime}$ satisfy the conditions of 3.0, $\mathscr{R}$ is the set of $\nu^{\prime}$ measureable sets, $\dot{\phi}$ is the $\mu \nu \mathscr{R}$-product measure, and if

$$
\nu^{\prime}<<\nu_{x}<<\nu^{\prime}
$$

for each $x \in M$, then

$.1 \phi$ is a p-inner regular borel measure, and

$.2 p$ is $\phi$-almost lindelof.

Proof. Let $\nu^{\prime \prime}(x, B)=\nu^{\prime}(B)$ for each $x \in M, B \in \mathscr{R}$. Then $\nu^{\prime \prime}$ is a regular conditional measure which is constant in $x$ and therefore continuous. Let $\phi^{\prime}$ be the (Elliott) $\mu \nu^{\prime \prime} \mathscr{R}$-product measure and complete the proof in Parts V, VI and VII below.

\footnotetext{
${ }^{2}$ Many of the results that follow hold also for non-finite measures.
} 
Part I. $\phi(A)=0 \leftrightarrow \phi^{\prime}(A)=0$

$\leftrightarrow A$ is a $\mu \nu$-nilset $\leftrightarrow A$ is a $\mu \nu^{\prime \prime}$-nilset.

Proof. $\phi(A)=0$

$\leftrightarrow A$ is a $\mu \nu$-nilset

(by $2.2 .4, .3$ )

$\leftrightarrow \iint C r_{A}(x, y) \nu_{x} d y \mu d x=0$

$\leftrightarrow \nu_{x}\left(A_{x}\right)=0$ for $\mu$-almost all $x \in M$, where $A_{x}=\{y \mid(x, y) \in A\}$

$\leftrightarrow \nu^{\prime}\left(A_{x}\right)=0$ for $\mu$-almost all $x$ (since $\left.\nu^{\prime}<<\nu<<\nu^{\prime}\right)$

$\leftrightarrow \nu_{x}^{\prime \prime}\left(A_{x}\right)=0$ for $\mu$-almost all $x$

$\leftrightarrow \iint C r_{A}(x, y) \nu_{x}^{\prime \prime} d y \mu d x=0$

$\leftrightarrow A$ is a $\mu \nu^{\prime \prime}$-nilset

$\leftrightarrow \phi^{\prime}(A)=0$.

Part II. $\quad \dot{\phi}^{\prime}<<\phi<<\phi^{\prime}$

Proof. Use Part I.

Part. III. If $A \in p$ (an open set) then for some countable subfamily $G$ of $m n$-open rectangles, $\sigma G \leqq A$, and $\phi^{\prime}(A-\sigma G)=0$.

Proof. This is Th. 2.2.3 of [4].

Part IV. $m n$-open rectangles are $\phi$-measurable.

Proof. This follows from 2.2.2.

Part V. $\phi$ is a borel measure.

Proof. Let $A \in p$. By Parts III and IV we can find a family $G$ for which $\sigma G \subseteq A, \sigma G$ is $\phi$-measurable and $\phi^{\prime}(A-\sigma G)=0$. But by Part I, $\dot{\phi}(A-\sigma G)=0$, and hence $A$ is $\phi$-measurable.

Part VI. $\quad p$ is $\phi$-almost lindelof.

Proof. Use 3.2.2 and Part I.

Part VII. $\phi$ is $p$-inner regular.

Proof. Let $A \in p$ and $\varepsilon>0$. Check that $\phi^{\prime}(A) \leqq \mu(M) \cdot \nu^{\prime}(N)<\infty$, and use 3.2.1 to secure such a sequence $c$ of $p$-closed sets that $c_{i} \subseteq$ $c_{i+1} \subseteq A$, and

$$
\phi^{\prime}(A)=\lim _{i \rightarrow \infty} \phi^{\prime}\left(c_{i}\right)
$$


Let $C=\bigcup_{i \in \omega} c_{i}$, and using Parts I and V, observe that $\phi^{\prime}(A-C)=0$, $\phi(A-C)=0, \phi(A)=\lim _{i \rightarrow \infty} \phi\left(c_{i}\right)$.

Consequently, $\phi$ is $p$-inner regular.

It is interesting to consider whether the conclusions of Theorem 3.3 remain valid when the condition $\nu^{\prime}\left\langle\left\langle\nu_{x}\left\langle\left\langle\nu^{\prime}\right.\right.\right.\right.$ is lessened to $\nu_{x}<<\nu^{\prime}$, or when this condition is removed altogether. The authors have been unable to settle these questions.

Theorems 3.4 and 3.5 below are further results in the spirit of 3.2 and 3.3, in which the continuity hypothesis has been replaced by a "separation of variables" condition.

THEOREM 3.4. If $\mu$ and $\nu$ satisfy conditions 3.0, and $\phi$ is the $\mu \nu \mathscr{R}$-product measure, and if there exist a $\mu$-integrable function $f$ and a measure $\psi$ on $N$ such that

$$
\nu(x, b)=f(x) \cdot \psi(b)
$$

for each $x \in M$ and $b \in \mathscr{R}$, then

$.1 \phi$ is a p-inner regular borel measure on $P$, and

$.2 p$ is $\phi$-almost lindelof.

Proof. For each set $C$, let

$$
C_{x}=\{y \mid(x, y) \in C\} \text {. }
$$

If $f(x)=0$ for each $x \in M$, or if $\psi(N)=0$, then the conclusions hold trivially. So we assume that $f\left(x_{0}\right)>0$ for some $x_{0} \in M$ and that $f(x)<\infty$ for all $x \in M$. Hence $\psi(b)=\nu\left(x_{0}, b\right) / f\left(x_{0}\right)$, for all $b \in \mathscr{R}$, and by 3.0.4, we conclude that $\psi$ is an $n$-inner regular borel measure and $n$ is $\psi$ almost lindelof. Let $G$ be the family of $\mu$-measurable sets, and for each $A \in G$, let

$$
h(A)=\int_{A} f(x) \mu d x \text { and } \gamma=m s s h M G .
$$

This defines a measure $\gamma$ on $M$, and $\gamma(A)=h(A)$ for $A \in G$. Since $\psi$ is $m$-continuous (indeed it is constant on $M$ ) we can define the $\gamma \psi \mathscr{R}$ product measure, $\phi^{\prime}$, and conclude from 3.2 that $\phi^{\prime}$ has the desired properties .1 and 2. We complete the proof by showing that $\phi^{\prime}=\phi$.

Let $F$ be the family of $\mu \nu \mathscr{R}$-basic rectangles and $\mu \nu$-nilsets, let $F^{\prime}$ be the family consisting of $\gamma \psi \mathscr{R}$-basic rectangles and $\gamma \psi$-nilsets, and let

$$
\begin{aligned}
g(C) & =\iint C r_{C}(x, y) \nu_{x} d y \mu d x, \\
g^{\prime}(C) & =\iint C r_{C}(x, y) \psi d y \gamma d x,
\end{aligned}
$$


for $C \in F$.

First, if $a \times b$ is a $\mu \nu \mathscr{R}$-basic rectangle, then

$$
\begin{aligned}
g^{\prime}(a \times b) & =\iint C r_{a \times b}(x, y) \psi d y \gamma d x \\
& =\psi(b) \cdot \gamma(a) \\
& =\psi(b) \int_{a} f(x) \mu d x \\
& =\int_{a} \nu(x, b) \mu d x \\
& =\int_{a} \int_{b} 1 \nu_{x} d y \mu d x \\
& =\iint_{C} C r_{a \times b}(x, y) \nu_{x} d y \mu d x \\
& =g(a \times b) .
\end{aligned}
$$

Secondly, if $C$ is a $\mu \nu$-nilset, then for $\mu$-almost all $x$,

$$
0=\int C r_{C}(x, y) \nu_{x} d y=\nu\left(x, C_{x}\right)=f(x) \cdot \psi\left(C_{x}\right),
$$

and hence,

$$
\begin{aligned}
g^{\prime}(C) & =\iint C r_{C}(x, y) \psi d y \gamma d x \\
& =\int \psi\left(C_{x}\right) \gamma d x \\
& =\int \psi\left(C_{x}\right) \cdot f(x) \mu d x \\
& =0=g(C) .
\end{aligned}
$$

Thus $g^{\prime}(C)=g(C)$, for $C \in F$.

Now let $Z=\{x \in M \mid f(x)=0\}$ and observe that $Z$ is $\mu$-measurable, and

$$
Z \times N \in F^{\prime} \text { and } g^{\prime}(Z \times N)=0 \text {. }
$$

Let

$$
F_{1}=\{(a \cup z) \times b \mid a \text { is } \mu \text {-measurable, } z \subseteq Z \text {, and } b \in \mathscr{R}\},
$$

and check that $F_{1}=F^{\prime}$.

Therefore,

$$
\begin{aligned}
\phi^{\prime} & =m s s g^{\prime} P F^{\prime} \\
& =m s s g^{\prime} P F_{1} \\
& =m s s g^{\prime} P F \\
& =m s s g P F \\
& =\phi .
\end{aligned}
$$


The result in 3.4 leads to the following

THEOREM 3.5. If $\mu$ and $\nu$ satisfy conditions 3.0 and $\phi$ is the $\mu \nu \mathscr{R}$-product measure, and if there exist, for each $i \in \omega, \mu$-integrable functions $f_{i}$ and measures $\psi_{i}$ on $N$ such that

$$
\nu(x, b)=\sum_{i \in \omega} f_{i}(x) \cdot \psi_{i}(b)
$$

for each $x \in M$ and $b \in \mathscr{R}$, then

$.1 \phi$ is a p-inner regular borel measure on $P$, and

$.2 p$ is $\phi$-almost lindelof.

Proof. For each $i \in \omega, x \in M$, and $b \in \mathscr{R}$, let

$$
\begin{aligned}
\nu_{i}(x, b) & =f_{i}(x) \cdot \psi_{i}(b), \\
\phi_{i} & =\left(\text { the } \mu \nu_{i} \mathscr{R} \text {-product measure }\right), \\
\phi^{\prime} & =\sum_{i \in \omega} \phi_{i} .
\end{aligned}
$$

By 3.4 learn that, for each $i \in \omega$,

$\phi_{i}$ is a $p$-inner regular borel measure on $P$, and $p$ is $\phi_{i}$-almost lindelof,

and hence, since these two properties carry over to countable sums, we have

$\phi^{\prime}$ is a $p$-inner regular borel measure on $P$, and $p$ is $\phi$-almost lindelof .

We complete the proof by showing that $\phi=\phi^{\prime}$.

Let $F$ be the family consisting of all $\mu \nu \mathscr{R}$-basic rectangles and $\mu \nu$-nilsets. For each $i \in \omega$ and $C \in F$, let

$$
\begin{aligned}
g_{i}(C) & =\iint C r_{C}(x, y) \nu_{i x} d y \mu d x \\
g(C) & =\iint C r_{C}(x, y) \nu_{x} d y \mu d x .
\end{aligned}
$$

Thus

$$
g(C)=\sum_{i \in \omega} g_{i}(C)
$$

for $C \in F$, and

$$
\begin{aligned}
\phi(A) & =m s s g(M \times N) F(A) \\
& =m s s\left(\sum_{i \in \omega} g_{i}\right)(M \times N) F(A)
\end{aligned}
$$




$$
\begin{aligned}
& =\sum_{i \in \omega} m s s g_{i}(M \times N) F(A) \\
& =\sum_{i \in \omega} \phi_{i}(A) \\
& =\phi^{\prime}(A),
\end{aligned}
$$

for $A \subseteq M \times N$. The third step above follows from Theorem 3.8 below and the fact that $F$ is disjunctive (See Definition 3.7), and the fact that the $g_{i}$ are nonnegative and countably additive on disjointed subsets of $F$.

The desired conclusion is at hand.

Questions that naturally arise are: when can a representation of the type (2) be obtained? How useful therefore is Theorem 3.5? No satisfactory answer to these questions is known to the authors at this time.

Theorem 3.2 (Elliott) generalizes Theorem 3.1 (Morse-Bledsoe) in yet another way, in that it uses one-sided nilsets $C$, where

$$
\iint C r_{C}(x, y) \nu_{x} d y \mu d x=0,
$$

instead of two-sided nilsets $D$, where

$$
\iint C r_{D}(x, y) \nu d y \mu d x=0=\iint C r_{D}(x, y) \mu d x \nu d y \text {. }
$$

Thus Theorem 3.1 is equally valid if the definition of $\mu \nu$-nilset given in $\S 2$ is amended to read: a subset $D$ of $M \times N$ is a $\mu \nu$-left nilset if

$$
\iint C r_{D}(x, y) \nu d y \mu d x=0 \text {. }
$$

Similarly, we could use $\mu \nu$-right nilsets.

The remainder of the paper gives results needed in the proof of Theorem 3.5.

LEMMA 3.6. If $T$ is a directed set with respect to the relation $\prec$ and if $0 \leqq A_{i t^{\prime}} \leqq A_{i t}$ whenever $i \in \omega, t \in T, t^{\prime} \in T$, and $t<t^{\prime}$, and if $\sum_{i \in \omega} A_{i t_{0}}<\infty$ for some $t_{0} \in T$, then

$$
\sum_{i \in \omega} \inf _{t \in T} A_{i t}=\inf _{t \in T} \sum_{i \in \omega} A_{i t} \text {. }
$$

Proof. Let $\varepsilon>0$ and select $N \in \omega$ so that

$$
\sum_{i=N}^{\infty} A_{i t 0}<\varepsilon / 2
$$


and then choose $\bar{t} \in T$ so that

$$
A_{i t} \leqq \inf _{t^{\prime} \in T} A_{i t^{\prime}}+\frac{\varepsilon}{2 N}, \text { for } \bar{t} \prec t, i<N .
$$

Thus for $\bar{t} \prec t, t_{0} \prec t$, we have

$$
\begin{aligned}
\sum_{i \in \omega} A_{i t} & =\sum_{i=0}^{N-1} A_{i t}+\sum_{i=N}^{\infty} A_{i t} \\
& \leqq \sum_{i=0}^{N-1} A_{i t}+\sum_{i=N}^{\infty} A_{i t_{0}} \\
& \leqq \sum_{i=0}^{N-1} A_{i t}+\varepsilon / 2 \\
& \leqq \sum_{i=0}^{N-1}\left(\inf _{t^{\prime} \in T} A_{i t^{\prime}}+\frac{\varepsilon}{2 N}\right)+\varepsilon / 2 \\
& =\sum_{i=1}^{N-1} \inf _{t^{\prime} \in T} A_{i t^{\prime}}+\varepsilon \\
& \leqq \sum_{i \in \omega} \inf _{t^{\prime} \in T} A_{i t^{\prime}}+\varepsilon .
\end{aligned}
$$

Therefore

$$
\inf _{t \in T} \sum_{i \in \omega} A_{i t} \leqq \sum_{i \in \omega} \inf _{t \in T} A_{i t}
$$

Since the reversed inequality is well known, the desired conclusion is at hand.

Definition 3.7. We say that a family $H$ is disjunctive if for each $G_{1}$ and each $G_{2}$ which are countable subfamilies of $H$, there is a countable pairwise disjointed subfamily $G$ of $H$ which is a refinement of both $G_{1}$ and $G_{2}$ and such that $\sigma G=\sigma G_{1} \cap \sigma G_{2}$.

\section{THEOREM 3.8. If}

$.1 H$ is disjunctive,

.2 for each $i \in \omega, g_{i} \geqq 0$,

$g_{i}$ is subadditive on $H$,

$g_{i}$ is countably additive on disjointed subfamilies of $H$,

$.3 A \subseteq S$, and

$.4 m s s\left(\sum_{i \in \omega} g_{i}\right) S H(A)<\infty$, then

$$
m s s\left(\sum_{i \in \omega} g_{i}\right) S H(A)=\sum_{i \in \omega} m s s g_{i S H}(A) .
$$

Proof. Let

${ }^{3}$ A function $f$ is said to be subadditive on $H$ if for each $B$ and each countable subfamily $G$ of $H$ for which $B \leqq \sigma G$, we have $f(B) \leqq \sum_{\alpha \in G} f(\alpha)$. 


$$
H_{A}=\{G \mid G \text { is a countable subfamily of } H \text { for which } A \subseteq \sigma G\} \text {. }
$$

Since $H$ is disjunctive it follows that $H_{A}$ is a directed set with respect to the refinement relation. Also using .2 it follows that

$$
0 \leqq \sum_{\alpha \in G^{\prime}} g_{i}(\alpha) \leqq \sum_{\alpha \in G} g_{i}(\alpha)
$$

whenever $i \in \omega, G \in H_{A}, G^{\prime} \in H_{A}$, and $G^{\prime}$ is a disjointed refinement of $G$. Furthermore, from .4 we know that for some $G_{0} \in H_{A}$,

$$
\sum_{\alpha \in G_{0}} \sum_{i \in \omega} g_{i}(\alpha)<\infty \text {. }
$$

Thus by Lemma 3.6 (identifying $H_{A}$ with $T$, and $A_{i t}$ with $\sum_{\alpha \in G} g_{i}(\alpha)$ ), we conclude that

$$
\sum_{i \in \omega} \inf _{G \in H_{A}} \sum_{\alpha \in G} g_{i}(\alpha)=\inf _{G \in H_{A}} \sum_{i \in \omega} \sum_{\alpha \in G} g_{i}(\alpha) .
$$

Consequently,

$$
\begin{aligned}
\operatorname{mss}\left(\sum_{i \in \omega} g_{i}\right) S H(A) & =\inf _{G \in H_{A}} \sum_{\alpha \in G} \sum_{i \in \omega} g_{i}(\alpha) \\
& =\inf _{G \in H_{A}} \sum_{i \in \omega} \sum_{\alpha \in G} g_{i}(\alpha) \\
& =\sum_{i \in \omega} \inf _{G \in H_{A}} \sum_{\alpha \in G} g_{i}(\alpha) \\
& =\sum_{i \in \omega} m s s g_{i} S H(A) .
\end{aligned}
$$

\section{REFERENCES}

1. S. K. Berberian, Counterexamples in Haar Measure, Amer. Math. Monthly, 73 No. 4, part II, (1966), 135-140.

2. W. W. Bledsoe and A. P. Morse, Product measures, Trans. Amer. Math. Soc., 79 (1955), 173-215.

3. R. E. Edwards, A theory of Radon measures on locally compact spaces, Acta Math. 89 (1953), 133-164.

4. E. O. Elliott, Measures on product spaces, Trans. Amer. Math. Soc., 128 (1967), 379-388.

5. M. C. Godfrey and M. Sion, On product of Radon measures, Canad. Math. Bull. 12 (1969), 429-444.

6. J. H. Hall, Topological Product Measures, Masters thesis, University of Texas, Austin, Texas, August, 1967.

7. R. A. Johnson, On Product Measures and Fubini's Theorem in Locally Compact Spaces, Doctoral Dissertation, State University of Iowa, 1964.

8. M. Loéve, Probability Theory, Princeton, New Jersey, D. Van Nostrand, 1963.

9. M. Mahowald, On the measurability of functions in two variables, Proc. Amer. Math. Soc., 13 (1962), 410-411.

Received May 20, 1971.

The University of Texas

AND

Pepperdine University 



\section{PACIFIC JOURNAL OF MATHEMATICS}

\section{EDITORS}

\section{H. SAMELSON}

Stanford University

Stanford, California 94305

\section{R. HOBBY}

University of Washington

Seattle, Washington 98105

\section{J. DugundjI}

Department of Mathematics University of Southern California Los Angeles, California 90007

RICHARD ARENS

University of California

Los Angeles, California 90024

\section{ASSOCIATE EDITORS}
E. F. BECKENBACH
B. H. NeumanN
F. WOLF
K. YOSHIDA

\section{SUPPORTING INSTITUTIONS}

\author{
UNIVERSITY OF BRITISH COLUMBIA \\ CALIFORNIA INSTITUTE OF TECHNOLOGY \\ UNIVERSITY OF CALIFORNIA \\ MONTANA STATE UNIVERSITY \\ UNIVERSITY OF NEVADA \\ NEW MEXICO STATE UNIVERSITY \\ OREGON STATE UNIVERSITY \\ UNIVERSITY OF OREGON \\ OSAKA UNIVERSITY
}

\author{
UNIVERSITY OF SOUTHERN CALIFORNIA \\ STANFORD UNIVERSITY \\ UNIVERSITY OF TOKYO \\ UNIVERSITY OF UTAH \\ WASHINGTON STATE UNIVERSITY \\ UNIVERSITY OF WASHINGTON \\ AMERICAN MATHEMATICAL SOCIETY \\ NAVAL WEAPONS CENTER
}

The Supporting Institutions listed above contribute to the cost of publication of this Journal, but they are not owners or publishers and have no responsibility for its content or policies.

Mathematical papers intended for publication in the Pacific Journal of Mathematics should be in typed form or offset-reproduced, (not dittoed), double spaced with large margins. Underline Greek letters in red, German in green, and script in blue. The first paragraph or two must be capable of being used separately as a synopsis of the entire paper. The editorial "we" must not be used in the synopsis, and items of the bibliography should not be cited there unless absolutely necessary, in which case they must be identified by author and Journal, rather than by item number. Manuscripts, in duplicate if possible, may be sent to any one of the four editors. Please classify according to the scheme of Math. Rev. Index to Vol. 39. All other communications to the editors should be addressed to the managing editor, Richard Arens, University of California, Los Angeles, California, 90024.

50 reprints are provided free for each article; additional copies may be obtained at cost in multiples of 50 .

The Pacific Journal of Mathematics is published monthly. Effective with Volume 16 the price per volume (3 numbers) is $\$ 8.00$; single issues, $\$ 3.00$. Special price for current issues to individual faculty members of supporting institutions and to individual members of the American Mathematical Society: $\$ 4.00$ per volume; single issues $\$ 1.50$. Back numbers are available.

Subscriptions, orders for back numbers, and changes of address should be sent to Pacific Journal of Mathematics, 103 Highland Boulevard, Berkeley, California, 94708.

PUBLISHED BY PACIFIC JOURNAL OF MATHEMATICS, A NON-PROFIT CORPORATION

Printed at Kokusai Bunken Insatsusha (International Academic Printing Co., Ltd.), 270, 3-chome Totsuka-cho, Shinjuku-ku, Tokyo 160, Japan. 


\section{Pacific Journal of Mathematics}

\section{Vol. 42, No. $3 \quad$ March, 1972}

Catherine Bandle, Extensions of an inequality by Pólya and Schiffer for vibrating membranes ................................ 543

S. J. Bernau, Topologies on structure spaces of lattice groups.......... 557

Woodrow Wilson Bledsoe and Charles Edward Wilks, On Borel product measures .......................................

Eggert Briem and Murali Rao, Normpreserving extensions in subspaces of

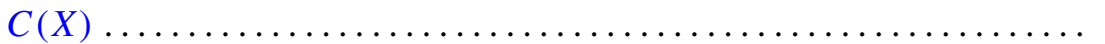

Alan Seymour Cover, Generalized continuation.................. 589

Larry Jean Cummings, Transformations of symmetric tensors .......... 603

Peter Michael Curran, Cohomology of finitely presented groups .......... 615

James B. Derr and N. P. Mukherjee, Generalized quasicenter and

hyperquasicenter of a finite group ...................... 621

Erik Maurice Ellentuck, Universal cosimple isols .................. 629

Benny Dan Evans, Boundary respecting maps of 3-mainfolds .......... 639

David F. Fraser, A probabilistic method for the rate of convergence to the

Dirichlet problem .................................. 657

Raymond Taylor Hoobler, Cohomology in the finite topology and Brauer

groups ..................................... 667

Louis Roberts Hunt, Locally holomorphic sets and the Levi form ........ 681

B. T. Y. Kwee, On absolute de la Vallée Poussin summability............ 689

Gérard Lallement, On nilpotency and residual finiteness in semigroups .... 693

George Edward Lang, Evaluation subgroups of factor spaces........... 701

Andy R. Magid, A separably closed ring with nonzero torsion pic ....... 711

Billy E. Rhoades, Commutants of some Hausdorff matrices ............. 715

Maxwell Alexander Rosenlicht, Canonical forms for local derivations . . . . 721

Cedric Felix Schubert, On a conjecture of L. B. Page ................ 733

Reinhard Schultz, Composition constructions on diffeomorphisms of $S^{p} \times S^{q}$

J. P. Singhal and H. M. (Hari Mohan) Srivastava, A class of bilateral generating functions for certain classical polynomials ....

Richard Alan Slocum, Using brick partitionings to establish conditions which insure that a Peano continuum is a 2-cell, a 2-sphere or an annulus...

James F. Smith, The p-classes of an $H^{*}$-algebra ...

Jack Williamson, Meromorphic functions with negative zeros and positive

poles and a theorem of Teichmuller ................. 